\title{
LA MUSICA E LO SVILUPPO LINGUISTICO IN ETÀ PRESCOLARE
}

\author{
Francesca Tinti \\ Sgrò Stefania \\ francescatinti8@gmail.com \\ Sapienza Università di Roma
}

Fecha de Recepción: 24 Febrero 2018

Fecha de Admisión: 10 Abril 2018

\section{SOMMARIO}

Le abilità ritmiche e lo sviluppo linguistico sono fortemente correlati ed evidenziabili già in età prescolare, nei bambini tra i 4 e i 6 anni.

II ritmo organizza eventi e gioca un ruolo fondamentale nella musica, nella fonologia e nella prosodia del linguaggio. Il linguaggio verbale come la musica è: inflessioni, intonazioni, tempo, ritmo e melodia. Bambini con difficoltà linguistiche hanno un'errata percezione della struttura ritmica; al contrario, con i Bambini che non presentano difficoltà linguistiche si evidenzia un alto grado di correlazione tra consapevolezza fonologica e abilità ritmiche, correlazione evidenziata da specifici test ritmici.

Parole chiave: musica; linguaggio; dislessia; abilità; sviluppo

\section{ABSTRACT}

\section{Music and linguistic development in prescular age.}

Rhythmic skills and linguistic development are strongly connected and already evident in preschool age for children between 4 and 6 years old.

The rhythm organizes events and plays a fundamental role in music, phonology and prosody of language. Verbal language and music are based on: inflections, intonations, time, rhythm and melody. Children with linguistic difficulties have a wrong perception of the rhythmic structure but on the other hand the children who don't present linguistic difficulties they present a high degree of correlation between phonological awareness and rhythmic abilities, connection highlighted by specific rhythmic tests.

Keywords: music; language; dyslexia; skills; development 


\section{LA MUSICA E LO SVILUPPO LINGUISTICO IN ETÀ PRESCOLARE}

\section{LA MUSICA, LO SVILUPPO COGNITIVO E LINGUISTICO}

La musica e un'attività complessa e molti studi, negli ultimi anni, hanno evidenziato che tocca non solo i sistemi uditivi e motori coinvolti nella percezione e nell' attività musicale, ma anche l'attenzione, l'integrazione multisensoriale, la memoria, l'apprendimento, il linguaggio, l'intelligenza sociale, la creatività e le emozioni.

Ascoltare e fare musica può influenzare i processi di sviluppo fisici, intellettivi, emotivi e sociali, (la capacita di imitare, le capacità motorie, le abilità sociali, l'alternarsi nei turni, il vocalizzare, il comportamento e di altri aspetti della comunicazione non verbale), connessi al concetto, proposto da Stern, di "sintonia dell'imitazione" (Stern, 1997). La musica è risultata essere efficace nel rafforzare lo spirito di gruppo e nel favorire la solidarietà. Indicativi sono stati anche i risultati ai test sociali nei quali si e dimostrato che i bambini delle scuole musicali sono più collaborativi di quelli delle scuole tradizionali e il numero degli alunni marginali oppure "esclusi" e nettamente inferiore. Anche le violenze tra bambini sono minori. Tra le varie ricadute positive del fare musica insieme e stato notato una riduzione del bullismo che, com'e noto, si sviluppa più facilmente quando vengono a mancare punti di riferimento, interessi, concentrazione, sensibilità, (Anna Oliverio Ferraris, 2004).

E ampiamente condivisa l'idea che lo studio di uno strumento musicale durante l'infanzia stimoli lo sviluppo cognitivo, aumentando le competenze del bambino anche in attività extramusicali come, per esempio, la lettura. Lo studio della musica comporterebbe un trasferimento dell'effetto di apprendimento dalle abilita specifiche, stimolate con l'attività musicale, alle funzioni cognitive di base (attenzione, discriminazione uditiva, memoria, coordinazione fine motoria, fantasia), necessarie per svolgere non solo i compiti musicali, ma anche extramusicali, quali la lettura e le abilita di calcolo a mente.

Musica e linguaggio sono fenomeni complessi che difficilmente possono essere studiati nell'insieme, generalmente vengono scomposti e analizzati nei vari elementi che li costituiscono. Ambedue utilizzano regole grammaticali e sintattiche che definiscono l'uso degli elementi di base: fonemi e parole nel linguaggio, note e accordi nella musica.

Corriveau e Goswami considerano centrale il ruolo della dimensione ritmico-temporale per una corretta conversione acustico-fonologica e quindi per lo sviluppo di un adeguato funzionamento dei processi di letto-scrittura (Flaugnacco e Lopez, 2014).

\section{IL LINGUAGGIO, LA MUSICA E GLI EFFETTI NEUROLOGICI}

Noi esseri umani, come specie, siamo creature musicali non meno che linguistiche. Siamo tutti in grado (con pochissime eccezioni) di percepire la musica: l'altezza delle note, il timbro, l'ampiezza degli intervalli, i contorni melodici, l'armonia e il ritmo. Noi integriamo tutto questo e "costruiamo" mentalmente la musica servendoci di molte parti diverse del cervello. II linguaggio verbale orale non e soltanto una successione di parole nell'ordine appropriato: ha inflessioni, intonazioni, tempo, ritmo e melodia. Linguaggio e musica dipendono entrambi da meccanismi di fonazione e articolazione che sono rudimentali negli altri primati; e anche per essere apprezzati e compresi, linguaggio e musica dipendono da meccanismi cerebrali distintamente umani, dedicati in modo specifico all'analisi di flussi di suoni complessi, segmentati e in rapido cambiamento (0. Sacks, 2010).

Molte ricerche sperimentali hanno evidenziato che la musica e in grado di stimolare i modelli interni del cervello favorendone l'impiego in ragionamenti complessi. I bambini con difficolta del linguaggio o con dislessia hanno spesso difficolta nel fare lo spelling, nel contare il numero delle sillabe 0 nel determinare se le parole fanno rima tra di loro. Tali difficolta sono constatate in lingue diverse anche se con sistemi di scrittura molto diversi.

Martina Huss e collaboratori hanno mostrato, usando una prova musicale che la dislessia e lega- 
ta ad una più accentuata difficolta nel percepire i ritmi o strutture ritmiche. I risultati hanno evidenziato una forte relazione fra abilita a percepire la struttura metrica della musica e l'apprendimento della lettura. In particolare, proprio l'abilita a percepire l'alternanza dei suoni (sillabe su cui cade 0 non cade l'accento) sarebbe importante per la corretta percezione del linguaggio. Essendo il ritmo più evidente nella musica e nel linguaggio, esporre i bambini a giochi musicali precoci potrebbe avere benefici nell'apprendimento (Huss M., V erney J.P ., Fosker T., Mead N., Goswami U. 2011).

\section{LA RICERCA}

\section{Obiettivi}

II presente studio di ricerca ha come fine la verifica dell'efficacia del corso propedeutico musicale come strumento preventivo, per i bambini in età prescolare, nei bambini dai 4 ai 6 anni.

\section{Metodologia}

Hanno partecipato alla ricerca 64 bambini in età prescolare, tutti provenienti dalla scuola d'infanzia nella periferia di Roma. II criterio di inclusione riguardava l'età: i bambini dovevano aver compiuto i 4 anni, mentre il criterio di esclusione riguardava: il ritardo mentale, la sordità e l'autismo. Sono stati somministrati, a tutti i bambini partecipanti al progetto di ricerca, i test per valutare le competenze ritmiche e linguistiche. I partecipanti alla ricerca sono stati suddivisi in due gruppi, un gruppo sperimentale e un gruppo di controllo. La durata del progetto è stata di 8 mesi, da dicembre (2015) a giugno (2016).

\section{Gli strumenti}

A tutti i bambini sono stati somministrati i seguenti test, per valutare le competenze ritmico/musicali e verbali:

1. II test Myra Stambak, prova di riproduzione ritmica e strutturale: valuta le capacità strutturoritmiche, fornisce informazioni sullo stato organizzativo-strutturale del bambino e sulle attività operative da scegliere in futuro. Sono state applicate valutazioni di livello quantitative.

2. II test Tinti, prova di riproduzione ritmica con accenti forti: valuta le capacità del bambino di riconoscere e riprodurre, in una semplice struttura ritmica, l'accento forte. La valutazione è quantitativa. II test permette di capire se il bambino possiede la capacità di discriminare l'alternanza di suoni (dove cadono o non cadono gli accenti).

3. La Batteria di Valutazione Neuropsicologica 5-11, prova sulle competenze verbali. In particolare è stata somministrata la Prova di fluente categoriale, che consiste nel chiedere ad ogni bambino di dire il maggior numero di animali, colori, frutti, in un minuto per ciascuna categoria, per un massimo di tre minuti.

\section{Fasi del progetto di ricerca}

Prima fase: somministrazione dei test al gruppo sperimentale e al gruppo di controllo;

Seconda fase: laboratorio di propedeutica sperimentale a cadenza settimanale per il gruppo sperimentale, per un periodo di quattro mesi;

Nella Terza fase sono stati somministrati, per una seconda volta i test utilizzati, sia al gruppo sperimentale e sia al gruppo di controllo;

Quarta fase: sono state verificate le ipotesi poste per la sperimentazione, con l'elaborazione di tutti i dati raccolti, mettendo a confronto le prestazioni del gruppo sperimentale e del gruppo di controllo ai test che valutano il ritmo e il linguaggio. 


\section{LA MUSICA E LO SVILUPPO LINGUISTICO IN ETÀ PRESCOLARE}

\subsection{Trattamento musicale: laboratorio propedeutico}

II trattamento si delinea come un laboratorio propedeutico musicale attivo a cadenza settimanale, per una durata di 45' per ciascuna classe appartenente al gruppo sperimentale. Gli obiettivi del laboratorio riguardavano:

Sensibilizzare le facoltà uditive in relazione ad un evento sonoro;

Controllare la voce e la respirazione;

Sviluppare le capacità ritmiche individualmente e di gruppo;

Porre attenzione all'ascolto di un brano musicale;

Controllare progressivamente l'utilizzo del movimento per accompagnare ed interpretare stimoli sonori e musiche.

II modello didattico-musicale utilizzato, per bambini in età prescolare, prevede un approccio metodologico e materiali che hanno come base comune la narrazione, elemento fondante l'attività nelle scuole dell'infanzia. II laboratorio propedeutico musicale per il gruppo sperimentale comprendeva la presentazione degli strumenti indicati di seguito:

La storia delle Note (appositamente sviluppata): è una breve storia, con lo scopo di presentare i 7 personaggi (le 7 note) e riporta esperienze ispirate ai vissuti 0 ai desideri dei bambini;

II libro dell'insegnante, punto di riferimento perché la lezione potesse avere delle linee guide da seguire;

La filastrocca-musicata delle Note: sottolinea le caratteristiche fisiche peculiari delle note rispetto al pentagramma, (Es: "il do fuori in giardino con grazioso cappellino, il Re quando va a letto la corona è nel cassetto...").

Le schede illustrate: offrono l'opportunità di avere un input percettivo-visivo rendendo stabili i concetti appresi con il canto e con la mimica accompagnata al canto.

II gioco con le marionette fa sì che si realizzi la storia e permette di agire la storia stessa, quindi viverla, invece che ascoltarla soltanto;

Gli esercizi e il gioco ritmico con gli strumenti hanno l'obiettivo di trovare uno spazio di comunicazione a un livello differente, cercare una ritmica comune o meno e di divertire imitando successioni ritmiche semplici e costruire delle sequenze gestuali da effettuare a tempo ( quello indicato dai compagni).

II gruppo di controllo, invece, svolgeva regolare attività didattica guidata dall'insegnante di riferimento.

\section{ANALISI DEI DATI}

Sui dati raccolti sono state applicate le statistiche descrittive. Per ogni gruppo (sperimentale e controllo) è stata rilevata la prestazione media prima e dopo il trattamento; attraverso il t-test si è ottenuto il confronto dei punteggi medi ottenuti alla prova di riproduzione ritmica (Stambak), alla prova di riproduzione ritmica con accenti forti (Tinti) e alla prova sulle competenze verbali (BVN).

È stato, inoltre misurato, l'effect-size standardizzato $d$ per confrontare il cambiamento tra le diverse prove.

Si è proceduto, in seguito, a stabilire il cut-off di miglioramento per ogni test (corrispondente alla differenza tra la media dei risultati ottenuti al post training meno la media ottenuta allo stesso test prima del training).

Infine è stata effettuata l'analisi delle correlazioni per indagare la relazione tra i tre test, pre e post trattamento. 
Tab.1. Gruppo sperimentale pre e post- intervento

\begin{tabular}{|c|c|c|c|c|c|}
\hline & $\mathrm{N}$ & Minimo & Massimo & Media & $\begin{array}{c}\text { Deviazione } \\
\text { std. }\end{array}$ \\
\hline Stambak pre & 32 & 0 & 15 & 4,03 & 3,797 \\
Stambak post & 32 & 1 & 15 & $\mathbf{8 , 8 1}$ & 4,638 \\
Tinti pre & 32 & 0 & 5 & 2,44 & 1,501 \\
Tinti post & 32 & 0 & 5 & $\mathbf{3 , 8 4}$ & 1,417 \\
BVN pre & 32 & 5 & 32 & 17,25 & 5,792 \\
BVN post & 32 & 11 & 30 & $\mathbf{2 0 , 0 0}$ & 4,799 \\
\hline
\end{tabular}

Tab.2. Gruppo di controllo pre e post- intervento

\begin{tabular}{|c|c|c|c|c|c|}
\hline & $\mathrm{N}$ & Minimo & Massimo & Media & $\begin{array}{c}\text { Deviazione } \\
\text { std. }\end{array}$ \\
\hline Stambak pre & 32 & 0 & 15 & 5,56 & 4,690 \\
Stambak post & 32 & 0 & 15 & $\mathbf{6 , 4 1}$ & 4,676 \\
Tinti pre & 32 & 0 & 5 & 2,19 & 1,693 \\
Tinti post & 32 & 0 & 5 & $\mathbf{2 , 9 1}$ & 1,692 \\
BVN pre & 32 & 1 & 32 & 18,41 & 7,343 \\
BVN post & 32 & 8 & 31 & $\mathbf{1 8 , 0 6}$ & 5,394 \\
\hline
\end{tabular}

\subsection{Analisi dei risultati ai test}

Attraverso i dati raccolti ed evidenziati dalla tab.1 e dalla tab.2 è stato possibile mettere a confronto le medie pre e post trattamento, ottenute ai test somministrati, del gruppo sperimentale e del gruppo di controllo, evidenziando un miglioramento più evidente del gruppo sperimentale.

Test Stambak: il gruppo sperimentale ha presentato, nel post-trattamento un incremento della media da 4.03 a 8.81. II gruppo di controllo, nel post trattamento, ha mostrato un incremento della media da 5.56 a 6.41 .

Test Tinti: per valutare l'incremento "reale" alla prestazione, risultato essere migliore nel gruppo sperimentale sin dalla prima somministrazione, è stata calcolata la differenza della media tra gruppo sperimentale e gruppo di controllo, il cui risultato è di 0.25 , sottratto alla differenza tra medie ottenute al retest, tra gruppo sperimentale e gruppo di controllo è di 0.93 . La discrepanza è stata ottenuta sottraendo a 0.93 lo 0.25 , ovvero: 0.68 . Possiamo dunque affermare che anche con il test ritmico Tinti, nel post trattamento, c'è stato un miglioramento più evidente del gruppo sperimentale rispetto a quello di controllo (punteggio 0.68).

Test linguistico BVN: il gruppo sperimentale ha presentato, nel post trattamento, un incremen- 


\section{LA MUSICA E LO SVILUPPO LINGUISTICO IN ETÀ PRESCOLARE}

to della media da 17.25 a 20.00. II gruppo di controllo, al test linguistico BVN, nel post trattamento ha mostrato un decremento della media da 18.41 a 18.06 .

Dai dati trattati emerge un miglioramento evidente nel gruppo sperimentale, in tutti e tre i test somministrati. II t test è stato utilizzato per spiegare un'eventuale differenza statistica nei punteggi medi ottenuti dai bambini che hanno completato i test al pre-trattamento e al post-trattamento, (tab. 3.1 e 3.2) Nel gruppo sperimentale i tre t test sono risultati tutti significativi $(p<.05)$.

Tab. 3.1 Effetto dell'intervento

\begin{tabular}{|c|c|c|c|c|c|c|}
\hline $\begin{array}{c}\text { Gruppo } \\
\text { Sperimentale }\end{array}$ & T-test & Sig. & Media & Dev. Std & Effect size & C.I. \\
\hline $\begin{array}{c}\text { Stambak1 vs } \\
\text { Stambak2 }\end{array}$ & $-7,087$ & 0 & $-4,781$ & 3,816 & 0,94 & $95 \%$ \\
\hline $\begin{array}{c}\text { Tinti1 vs } \\
\text { Tinti2 }\end{array}$ & $-5,464$ & 0 & $-1,406$ & 1,456 & 0,43 & $95 \%$ \\
\hline $\begin{array}{c}\text { BVN1 vs } \\
\text { BVN2 }\end{array}$ & $-4,603$ & 0 & $-2,750$ & 3,379 & 0,40 & $95 \%$ \\
\hline
\end{tabular}

Tab. 3.2 Effetto dell'intervento

\begin{tabular}{|c|c|c|c|c|c|c|}
\hline $\begin{array}{c}\text { Gruppo } \\
\text { controllo }\end{array}$ & T-test & Sig. & Media & Dev. Std & Effect size & C.I. \\
\hline $\begin{array}{c}\text { Stambak1 vs } \\
\text { Stambak2 }\end{array}$ & $-1,057$ & 0 & $-0,844$ & 4,516 & 0,94 & $95 \%$ \\
\hline $\begin{array}{c}\text { Tinti1 vs } \\
\text { Tinti2 }\end{array}$ & $-2,463$ & 0 & $-0,719$ & 1,651 & 0,43 & $95 \%$ \\
\hline $\begin{array}{c}\text { BVN1 vs } \\
\text { BVN2 }\end{array}$ & $-0,435$ & 0 & $-0,344$ & 4,469 & 0,40 & $95 \%$ \\
\hline
\end{tabular}

\subsection{Analisi individuali}

Dalle analisi effettuate sui due gruppi, sperimentale e controllo, emergono possibili valutazioni individuali, eseguite dopo il laboratorio propedeutico musicale (post-trattamento). Si è, dunque, proceduto a determinare il cut-off per ogni test somministrato al gruppo sperimentale (tab.7), valutando la differenza tra media dei risultati ottenuti al pre-trattamento e al post-trattamento, relativamente ai tre test somministrati, il test ritmico Stambak, il test ritmico Tinti e il test linguistico BVN. Si è proceduto a rilevare quanti, tra i partecipanti alla ricerca, mostravano un incremento nella prestazione, quanti hanno raggiunto o superato i valori di miglioramento (Tab. 7 )

Relativamente al test Stambak, rispetto ai cutoff $(4,78)$, emersi dai punteggi ottenuti ai test del gruppo sperimentale, si è rilevato un miglioramento nella prestazione dei bambini, ai test somministrati al post trattamento. In particolare, emerge che il $\mathbf{5 6 , 2 5 \%}$ dei bambini del gruppo sperimentale ha superato il cut-off stabilito. Nello specifico, 18 bambini $(n=32)$ del gruppo sperimentale hanno mostrato un miglioramento nella performance raggiungendo la soglia stabilita, (il superamento del cut-off è pari a punti: 6,22 per 1 bambino; 5,22 per 1 bambino; 4,22 per 5 bambini; 3,22 per 1 bambino; 2,22 per 3 bambini). Un bambino ha ottenuto il punteggio massimo (punteggio di 15), al pre e al post trattamento. Relativamente al gruppo di controllo è emerso che il $15 \%$ dei bambini ha superato il cut-off stabilito di 4,78 (superamento del cut-off pari a punti: 8,22 per 1 bambino; 4,22 per 1 bambino; 0,22 per 2 bambini). Nello specifico, 5 bambini $(n=32)$ del gruppo di controllo hanno mostrato rilevanti miglioramenti.

Relativamente al Test Tinti, (cut-off 1,4), emerge che il $46,87 \%$ dei bambini del gruppo speri- 
mentale ha superato il cut-off stabilito (superamento del cutoff pari a punti: 3,6 per 1 bambino; 2,6 per 2 bambini; 1,6 per 3 bambini; 0,6 per 9 bambini). Nello specifico, 15 bambini $(n=32)$ hanno mostrato un miglioramento nella performance raggiungendo la soglia stabilita. Invece, nel gruppo di controllo è emerso che il $\mathbf{1 8 , 7 5 \%}$ dei bambini ha superato il cut-off stabilito, di 1,4 (superamento del cutoff di: 3,6 per 1 bambino; 2,6 per 1 bambino; 0,6 per 3 bam- bini; 0,6 per 4 bambini). Nello specifico, 6 bambini $(n=32)$ hanno mostrato rilevanti miglioramenti nella performance raggiungendo il cut-off stabilito.

Rispetto al test BVN 5/11 (cut-off 2,75), emerge che il 50\% dei bambini del gruppo sperimentale ha superato il cut-off stabilito ( superamento del cut-off pari a punti: 6,25 per 1 bambino; 4,25 per 2 bambini; 3,25 per 3 bambini; 2,25 per 4 bambini; 1,25 per 4 bambini; 0,25 per 3 bambini). Nello specifico, 16 bambini $(n=32)$ hanno mostrato un miglioramento della performance raggiungendo la soglia stabilita e cinque bambini, valutando il loro pre e post trattamento, hanno mantenuto lo stesso punteggio. Relativamente ai punteggi ottenuti dal gruppo di controllo è emerso che il $18,75 \%$ dei bambini ha superato il cut-off stabilito di 2,75 ( superamento del cut-off di: 9,25 per 1 bambino; 3,25 per 1 bambino; 2,25 per 1 bambino; 1,25 per un bambino; 0,25 per 1 bambino). Nello specifico, 6 bambini $(n=32)$ hanno migliorato la performance raggiungendo il cut-off stabilito.

\section{ANALISI DELLE CORRELAZIONI}

L'analisi delle correlazioni ha permesso di verificare se i punteggi ottenuti ai tre test somministrati presentino una relazione lineare e statisticamente significativa, (tab. 4). Rispetto al campione totale $(n=64)$, l'analisi dei dati ha mostrato una correlazione positiva e statisticamente significativa con i punteggi ottenuti al test Stambak pre e i punteggi ottenuti al test Stambak post $(r=.491 ; p<$ $.01)$, con il test Tinti pre $(r=, 440 ; p<.01)$, con il BVN pre $(r=, 428 ; p<.01)$ e il BVN post $(r=, 370$; $\mathrm{p}<.01)$.

Emerge una correlazione positiva e statisticamente significativa tra il test Stambak post e il test Tinti pre $(r=, 365 ; p<.01)$ e il Tinti post $(r=, 576 ; p<.01)$; una relazione positiva e statisticamente significativa tra il test Tinti pre e il test Tinti post $(r=, 514 ; p<.01)$ e tra il test Tinti pre e il test BVN pre $(r=, 282 ; p<.05)$ e il test BVN post $(r=, 387 ; p<.01)$; inoltre, emerge una relazione positiva e statisticamente significativa tra il test BVN pre e il BVN post $(r=, 767 ; p<.01)$.

Rispetto al gruppo sperimentale $(n=32)$, i dati presentano una correlazione positiva e statisticamente significativa tra il test Stambak pre e il test Stambak post $(r=, 607 ; p<.01)$, il test Tinti pre $(r=$ $, 422 ; p<.05)$, il test BVN pre $(r=, 391 ; p<.05)$ e il test BVN post $(r=, 361 ; p<.05)$; il test Stambak post presenta una correlazione positiva e statisti- camente significativa con il test Tinti pre $(r=, 596$; $p<.01)$, il test Tinti post $(r=, 516 ; p<.01)$; emerge una correlazione positiva e statisticamente significativa con il test Tinti post $(r=, 503 ; p<.01)$ e il Test Tinti pre. II test BVN pre presenta una correlazione positiva e statisticamente significativa con il test BVN post $(r=, 812 ; p<.01)$.

Rispetto al gruppo di controllo emerge una correlazione positiva e statisticamente si- gnificativa tra il test Stambak pre e i seguenti test: test Stambak post $(r=, 535 ; p<.01)$, il test Tinti pre $(r=$ $, 494 ; p<.01)$, il test BVN pre $(r=, 440 ; p<.05)$, il BVN post $(r=, 460 ; p<.01)$; il test Stambak post presenta una correlazione positiva e statisticamente significativa con il test Tinti post $(r=, 568 ; p<.01)$. II test Tinti pre-trattamento evidenzia una correlazione positiva e statisticamente significativa con: il test Tinti post $(r=, 524 ; p<.01)$, il test BVN pre $(r=, 370 ; p<.05)$, il BVN post $(r=, 405 ; p<.05)$; infine, emerge una correlazione positiva e statisticamente significativa tra il test BVN pre con il test BVN post $(r=, 796 ; p<.01)$. 


\section{LA MUSICA E LO SVILUPPO LINGUISTICO IN ETÀ PRESCOLARE}

Tab. 4. Correlazioni gruppo sperimentale

\begin{tabular}{|c|c|c|c|c|c|c|c|}
\hline & & Stambak1 & Stambak2 & Tinti1 & Tinti2 & BVN1 & BVN2 \\
\hline \multirow[t]{3}{*}{ Stambak1 } & Correlazione di Pearson & 1 & $.607^{m / n}$ & .422 & .271 & .391 & $.361^{\prime \prime}$ \\
\hline & Sig. (2-code) & & , & .016 & .134 & .027 & 042 \\
\hline & $\mathbf{N}$ & 32 & 32 & 32 & 32 & 32 & 32 \\
\hline \multirow[t]{3}{*}{ Stambak? } & Gorrelazione al Pearson & .607 & 1 & $.596^{\circ}$ & $.516^{-1}$ & .032 & .216 \\
\hline & Sig. (2-code) & (ם & & ס0ם. & .003 & 863 & .235 \\
\hline & $\mathbf{N}$ & 32 & 32 & 32 & 32 & 32 & 32 \\
\hline \multirow[t]{3}{*}{ Tinti1 } & Correlazione di Pearson & $.422^{\prime \prime}$ & $.595^{\mathrm{Nm}}$ & 1 & $.503^{-11}$ & .180 & .349 \\
\hline & Sig. (2-code) & .016 &, 000 & & .003 & , 324 & 050 \\
\hline & $\mathbf{N}$ & 32 & 32 & 32 & 32 & 32 & 32 \\
\hline \multirow[t]{3}{*}{ Tirnti2 } & Correlazione di Pearson & .271 & $.515^{10}$ & $.503^{n+m}$ & 1 & -.023 & .033 \\
\hline & Sig. (2-code $)$ & .134 & .003 & .003 & & .802 & .857 \\
\hline & $\mathbf{N}$ & 32 & 32 & 32 & 32 & 32 & 32 \\
\hline \multirow[t]{3}{*}{ BVN1 } & Correlazione di Pearson & 391 & ,032 & $.1 \mathrm{BD}$ & -.023 & 1 & 812" \\
\hline & Sig. (2-code) & .027 & .663 & 324 & .902 & & .000 \\
\hline & N & 32 & 32 & 32 & 32 & 32 & 32 \\
\hline \multirow[t]{3}{*}{ BVN2 } & Correlazione di Pearson & $.361^{\prime \prime}$ & .216 & .349 & .033 & .812 & 1 \\
\hline & Sig. (2-code) & .042 & .235 & .050 & .057 & .000 & \\
\hline & $\mathbf{N}$ & 32 & 32 & 32 & 32 & 32 & 32 \\
\hline
\end{tabular}

*. La correlazione è significativa al livello 0,01 (2-code)

\section{CONCLUSIONI}

Questo studio conferma le precedenti ricerche che evidenziavano una relazione tra abilità ritmiche e sviluppo linguistico. II miglioramento del gruppo sperimentale, che ai test somministrati ha ottenuto punteggi elevati, è più evidente rispetto al gruppo di controllo: il 56,25\%; dei bambini del gruppo sperimentale, dopo aver partecipato al laboratorio propedeutico musicale, presenta un notevole miglioramento nella prova di riproduzione ritmica e strutturale, superando la soglia del punteggio stabilito, rispetto al $15 \%$ dei bambini del gruppo di controllo.

Lo stesso miglioramento, il 46,87\% dei bambini del gruppo sperimentale, si evidenzia anche nelle capacità di riconoscere e riprodurre l'accento forte in una stessa struttura ritmica (Test Tinti), al contrario pochi bambini del gruppo di controllo hanno raggiunto un miglioramento. Infine, un dato rilevante è emerso dalla somministrazione della Batteria di valutazione neuropsicologica: il $50 \%$ dei bambini del gruppo sperimentale, nella prova sulle competenze verbali, ha evidenziato un notevole miglioramento nella prova di fluente categoriale, invece, solo il 18\% dei bambini del gruppo di controllo ha raggiunto il cut-off di miglioramento del 2.75.

In conclusione, i diversi studi effettuati in precedenza e la presente ricerca suggeriscono che una formazione musicale può dare beneficio ai bambini, nell'ambito dello sviluppo linguistico, in età prescolare.

Nonostante si possano evidenziare delle limitazioni nello studio effettuato, che riguardano un ristretto campo di valutazione delle competenze cognitive del bambino, l'aspetto positivo è stato riscontrato nella facilità della somministrazione e nella analisi dei punteggi dei test. Infatti, sia i due test ritmici (Stambak e Tinti) che il test linguistico (BVN) sono brevi (massimo 6 minuti a bambino), semplici (sia da spiegare che da somministrare) e adeguati all'età (4 - 6 anni).

In conclusione si è potuto rilevare come, bambini che abbiano ottenuto punteggi elevati allo Stambak pre-trattamento, abbiano anche mostrato punteggi altrettanto elevati sugli altri test (il test ritmico Tinti e il test linguistico BVN).

Per approfondimenti, in futuro, si potrebbero valutare ulteriori competenze acquisite dal bambino in età prescolare dopo un trattamento musicale e rendere più partecipi e attive le insegnanti, sia in classe durante il trattamento musicale sia attraverso la compilazione di un questionario valutativo del bambino. 


\section{BIBLIOGRAFIA}

Aniruddh D. Patel, John R. Iverson (2007). "The linguistic benefits of musical abilities", Trends in Cognitive Sciences.

Anvari, S. H., Trainor, L. J., Woodside, J., and Levy, B. A. (2002). Relations among musical skills, phonological processing, and early reading ability in preschool children. J. Exp. Child Psychol. 83, 111-130.

Benton A.L., 1987:"La musica e il Cervello", Padova: Piccin.

Bunt L. (1997): Music Therapy An Art Beyond Words.

Cornoldi, C., and Tressoldi, P. (2007). "Definizione, criteri e classificazione," in Difficoltà e Disturbi Dell'apprendimento, ed C. Cornoldi (Bologna: Il Mulino), 9-52.

Corriveau K.H., Goswami U., (2009). Rhythmic motor entrainment in children with speech and language impairments: tapping to the beat. Cortex 45, 2009, 119-130.

De Martino, S., Espesser, R., Rey V., Habib, M. (2001). The temporal processing deficit hypothesis in dyslexia: new experimental evidence. Brain Cogn. 46, 104-108.

Goswami, U., Huss, M., Mead, N., Fosker, T., and Verney, J. P. (2013). Perception of patterns of musical beat distribution in phonological developmental dyslexia: significant longitudinal relations with word reading and reading comprehension. Cortex 49, 1363-1376. doi: 10.1016/.

Hans B., (1998) : Crescere con la Musica.

Musacchia G., (2011): "Lo studio della musica potenzia le capacità verball”, rivista Americana delle Scienze, PNAS.

Peretz I., Coltheart M. (2003), "Modularity of music processing”, Nature Neuroscience, 6 (7), 688$691)$.

Sacks 0., 2007: "Musicofilia”, Gli Adelphi.

Shaw, Gordon, (1999): "Keeping Mozart in Mind".

Stern D., 1977: The first relatioship: Infant and Mother, Londra: Fontana

Thomson, J. M., and Goswami, U. (2008). Rhythmic processing in children with developmental dyslexia: auditory and motor rhythms link to reading and spelling. J. Physiol. 102, 120-129. 
